\title{
High range of motion in the first ten postoperative days after TKA does not predict superior outcome in the long run
}

\author{
Paul Köglberger ${ }^{1} \cdot$ Alexander Wurm $^{2} \cdot$ Débora Coraça-Huber $^{3} \cdot$ Martin Krismer $^{2} \cdot$ Wilhelm Oberaigner $^{4}$. \\ Michael Liebensteiner ${ }^{2}$
}

Received: 17 April 2020 / Accepted: 17 March 2021 / Published online: 25 March 2021

(c) The Author(s) 2021

\begin{abstract}
Introduction To retrospectively investigate the early postoperative range of motion (ROM) (days 4, 7, 10) after total knee arthroplasty (TKA) and to test for associations (a) with long-term outcome in terms of ROM and (b) with a disease-specific knee score.

Materials and methods A retrospective analysis was performed in patients with previous primary TKA. Data taken from the medical records were ROM from preoperative and postoperative days 4, 7 and 10 and 1 year. As patient-reported outcome the Western Ontario and McMaster Universities Osteoarthritis Index (WOMAC Score) was taken from preoperative and one year after TKA.

Results 316 patients (330 knees) were available. Only negligible correlations were determined between ROM at twelve months postoperative and ROM in the early postoperative days (days $4,7,10$ ). Similarly, only negligible correlations were determined between ROM in the early postoperative days (days 4, 7, 10) and the 1-year WOMAC.

Conclusion From the main findings it would seem that steepness of ROM ascent in the early postoperative days is of minor importance for (a) long-term ROM and (b) long-term knee score outcome after TKA.
\end{abstract}

Keywords Total knee arthroplasty $\cdot$ Range of motion $\cdot$ Fast track $\cdot$ Rapid recovery $\cdot$ Knee replacement

$\begin{array}{ll}\text { Abbreviations } & \\ \text { ROM } & \text { Postoperative range of motion } \\ \text { SPSS } & \begin{array}{l}\text { Statistical Package for the Social } \\ \text { Sciences }\end{array} \\ \text { TKA } & \text { Total knee arthroplasty } \\ \text { WOMAC Score } & \begin{array}{l}\text { Western Ontario and McMaster Univer- } \\ \text { sities Osteoarthritis Index Score }\end{array}\end{array}$

Alexander Wurm

alexander.wurm@i-med.ac.at

1 Medical University of Innsbruck, Innsbruck, Austria

2 Department of Orthopaedics and Traumatology, Medical University of Innsbruck, Innsbruck, Austria

3 Department of Experimental Orthopaedics, Medical University of Innsbruck, Innsbruck, Austria

4 Department of Clinical Epidemiology, Tyrolean Federal Institute for Integrated Care, Tirol Kliniken $\mathrm{GmbH}$, Innsbruck, Austria

\section{Introduction}

In daily clinical practice the question often arises as to when to discharge a patient after routine total knee arthroplasty (TKA). The fact that there is no generally accepted consensus on this question is also reflected by the length of hospital stay, which varies in general between two and 15 days [1-13] with even longer stays observed [14, 15]. Besides other clinical Landmarks like wound drainage or pain control also the progression of early postop range of motion (ROM) may be considered [4, 7, 8, 13, 16]. With regard to ROM it might particularly be speculated whether early postoperative ROM (postoperative day 1-4) is a good indicator of long-term postoperative ROM. Moreover, it might also be speculated whether early postoperative ROM is associated with general outcome after TKA. In such a case it would be useful to accelerate the gain in early ROM immediately after TKA, as seen in many fast-track programs following TKA. Moreover, it would also be justified to use ,early ROM' for the decision on when and where to discharge a patient from hospital as well as for identifying those at high risk for poor long-term outcome after TKA. 
Regarding associations between early postoperative ROM and long-term ROM after TKA, Bade et al. found that knee flexion at postoperative day 2 was not related to knee flexion at six months postoperative [3]. Others found that greater knee flexion at discharge did not predict knee flexion one year postoperative [17]. Regarding associations between early post-op ROM and long-term knee score outcome after TKA, previous authors reported that the amount of knee flexion (and extension) at discharge after TKA was not associated with the Oxford knee score at one year postoperative [17]. Aside from the above-mentioned two articles, the available evidence is rare.

Based on the above-mentioned lack of evidence it was the aim of the study to test whether early postop ROM (days 4, $7,10)$ was associated with ROM at 12 months postoperative (Hypothesis 1) or with the WOMAC Score at 12 months postoperative (Hypothesis 2).

\section{Materials and methods}

The study design was retrospective observational. Data already available from clinical routine were analyzed after approval by the ethics committee of the Medical University. Consecutive patients who previously underwent primary TKA as part of the clinical routine were analyzed. Cases were excluded in the case of: (a) incomplete WOMAC data, (b) incomplete ROM data, (c) primary prostheses other than cruciate-retaining. Included were patients with Triathlon CR, Scorpio CR and Scorpio NRG CR implants (Stryker, Kalamazoo, MI, USA).

During the study period (January 2005 to August 2015), 2093 TKA were performed at our institution. Of those, 988 had to be excluded because either the preoperative or postoperative or both WOMAC Scores were incomplete. Of the remaining 1105 TKA 242 were excluded because other implants than above-mentioned CR implants had been used. Of the remaining 863 primary TKA only 330 cases could be included in the final analysis. The rest had to be excluded because no information on ROM was available.

ROM data collected with goniometers were taken from the medical records for the following points in time: preoperative, postoperative days 4,7 and 10 and 1 year ( \pm 6 months) postoperative. The early postoperative measurements were taken from the obligatory daily ROM documentation of our physiotherapists. The one-year ROM was taken from the documentation of the one-year outpatient visit.

For patient-reported outcome measurement the Western Ontario and McMaster Universities Osteoarthritis Index (WOMAC) was applied in the German-language version [18]. The questionnaire was completed shortly before surgery during the hospital stay (preoperatively) and postoperatively the questionnaire was sent directly to the patient one year after surgery. The WOMAC questionnaire collects data on pain, stiffness, and physical function. Every item was completed on an 11-point scale and converted for analysis purposes to a scale from 0 to 100,0 denoting the best and 100 the worst response. The score for each of the three main dimensions is defined as the sum of all item scores divided by the number of items. The total score was defined as the sum of pain, stiffness and function scores divided by three.

Data were extracted from the records and stored in spreadsheet format. Further analyses were performed with SPSS. As descriptive statistics medians and inter-quartile-ranges were determined. Kolmogorov-Smirnov tests indicated that the data sets were not normally distributed. Therefore, the above-mentioned Hypotheses 1-4 were tested with Spearman correlation coefficients; alpha was defined as 0.05 . With regard to Spearman's correlation coefficient, the size of the correlation was assumed as very high from 0.90 to 1.00 , high from 0.70 to 0.90 , moderate from 0.50 to 0.70 , low from 0.30 to 0.50 and negligible from 0.00 to 0.30 [19].

\section{Results}

316 patients (330 knees; 140 left, 190 right) with a median age of 70 years (IQR 12) were available for analysis.

Knee ROM (Medians \pm Interquartile-Ranges) changed from $110 \pm 20^{\circ}$ preoperative to $65 \pm 20^{\circ}$ on day 4 , further to $85 \pm 15^{\circ}$ on day 7 and $90 \pm 15^{\circ}$ on day 10 . Twelve months after surgery ROM was $110 \pm 20^{\circ}$. The total WOMAC Score was $52 \pm 28$ preoperative and changed to $16 \pm 29$ at 12 months postoperative (Table 1).

Regarding Hypothesis 1, negligible correlations were determined between ROM at twelve months postoperative and ROM in the early postoperative days (days 4, 7, 10) (Table 2).

Similarly, regarding Hypothesis 2, only negligible correlations were determined between ROM in the early postoperative days (days $4,7,10$ ) and the 12-month WOMAC outcome (Table 3 ).

\section{Discussion}

The most important finding made in the study was that the ROM obtained in the early postoperative days (days 4, 7, 10) showed only negligible correlations with the WOMAC score at 12 months postoperative and ROM at 12 months postoperative.

When attempting to compare our findings with previous research, it became obvious that evidence on this specific issue is scarce. Regarding the influence of early postoperative ROM on long-term postoperative ROM, supporting evidence comes from Bade et al., who found that knee flexion 
Table 1 Descriptive statistics of ROM and knee score data

\begin{tabular}{lll}
\hline & Median & IQR \\
\hline ROM [ $\left.{ }^{\circ}\right]$ & & \\
Preoperative & 110 & 20 \\
Day 4 & 65 & 20 \\
Day 7 & 85 & 15 \\
Day 10 & 90 & 15 \\
12 months & 110 & 20 \\
WOMAC total & & \\
Preoperative & 52 & 28 \\
12 months & 16 & 29 \\
WOMAC pain & & 30 \\
Preoperative & 50 & 28 \\
12 months & 12 & \\
WOMAC stiffness & & 40 \\
Preoperative & 55 & 30 \\
12 months & 20 & 32 \\
WOMAC function & & \\
Preoperative & 54 & \\
12 months & 16 & \\
\hline
\end{tabular}

Table 2 Correlation analysis between ROM after 12 months postoperative and in the early postoperative days

\begin{tabular}{cl}
\hline & $\begin{array}{l}\text { ROM 12 months } \\
\text { coefficient }\end{array}$ \\
& $p$ value \\
\hline ROM & \\
Day 4 & 0.105 \\
& 0.097 \\
Day 7 & 0.219 \\
& $0.000^{*}$ \\
Day 10 & 0.243 \\
& $0.001 *$ \\
\hline
\end{tabular}

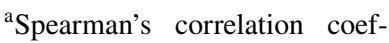
ficient

*Significant correlation at the 0.05 level (2-tailed)

at postoperative day $2 \mathrm{did}$ not influence knee flexion at six months postoperative [3]. Our findings are also in good agreement with those obtained by Naylor et al. who reported that knee flexion at discharge did not influence the amount of knee flexion at one year postoperative [17]. However, in contrast to the 330 cases in our study, the above-mentioned previous studies presented 64 [3] and 133 cases [17].

With regard to the influence of early postoperative ROM on long-term postoperative knee score outcome, previous researchers tested for associations between knee flexion at discharge and the Oxford knee score one year postoperative [17]. They found that neither maximum knee flexion nor maximum knee extension at discharge had an influence on
Table 3 Correlation analysis between ROM in the early postoperative days and the 12-month WOMAC score outcome

\begin{tabular}{|c|c|c|c|c|}
\hline & $\begin{array}{l}\text { WOMAC } \\
\text { total } \\
12 \text { months }\end{array}$ & $\begin{array}{l}\text { WOMAC } \\
\text { pain } \\
12 \text { months }\end{array}$ & $\begin{array}{l}\text { WOMAC } \\
\text { stiffness } \\
12 \text { months }\end{array}$ & $\begin{array}{l}\text { WOMAC } \\
\text { function } \\
12 \text { months }\end{array}$ \\
\hline & Coefficienta & Coefficienta & Coefficienta & Coefficienta \\
\hline & $p$ value & $p$ value & $p$ value & $p$ value \\
\hline \multicolumn{5}{|l|}{ ROM } \\
\hline \multirow[t]{2}{*}{ Day 4} & -0.038 & -0.031 & -0.044 & -0.039 \\
\hline & 0.501 & 0.590 & 0.434 & 0.489 \\
\hline \multirow[t]{2}{*}{ Day 7} & -0.097 & -0.059 & -0.112 & -0.107 \\
\hline & 0.086 & 0.295 & $0.048 *$ & 0.058 \\
\hline \multirow[t]{2}{*}{ Day 10} & -0.073 & -0.040 & -0.110 & -0.066 \\
\hline & 0.255 & 0.534 & 0.086 & 0.310 \\
\hline
\end{tabular}

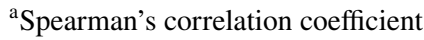

*Significant correlation at the 0.05 level (2-tailed)

the Oxford knee score one year postoperative. This is in good agreement with the findings made in our study. We were not able to find other publications dealing with the above-mentioned specific research question.

Our findings are regarded as highly clinically relevant. Due to the fact that early postoperative ROM did not predict long-term knee score outcome or long-term ROM after TKA, it does not seem necessary to force ROM gain too strongly in the initial postoperative days. Probably, preoperative ROM and proper biomechanics of the TKA are predictors of much greater importance than is steepness of ROM ascent in the early postoperative period. Fast-track rehabilitation programs that aim to substantially accelerate ROM gain in the early postoperative period might also be questioned. Although the findings from the current study support the practice of not forcing ROM gain too aggressively in first postoperative weeks there should be a limit to this in patients that do not achieve a minimum of $90^{\circ}$ knee flexion within the first postoperative weeks. Yao et al. demonstrated that these patients can successfully be treated with early manipulating under anesthesia. [20]

Other researchers investigated the effect of preoperative ROM instead early-postoperative ROM on long-term ROM outcome. The preoperative maximum knee flexion angle was found positively correlated with the flexion angles at 6 months $[3,5,21,22]$ and 12 months [9, 17, 22-24]. Beside the influence of many other variables on the postoperative range of flexion, greater preoperative flexion angle was considered to have the most positive effect on the long-term flexion achieved after surgery and therefore the preoperative flexion was considered to be the primary determining variable of the long-term flexion outcome after TKA [22, 24]. Others measured preoperative ROM instead the flexion angle and reported that the preoperative ROM 
was significantly correlated with ROM at 6 months [25] and 12 months $[25,26]$. When counting both studies reporting ROM and maximum knee flexion angle there seems to be a strong evidence for the relationship between preoperative and long-term postoperative ROM.

Trying to find publications on a potential relationship between preoperative ROM and long-term postoperative knee scores, it appears that there are only few studies available. Naylor et al. reported that the preoperative maximum knee flexion angle was not a significant predictor of the Oxford Knee Score at 1-year postoperative [17]. Similarly, the preoperative maximum knee extension angle did not significantly predict the Oxford Knee Score at 1-year postoperatively [17]. However, others found that preoperative ROM was positively correlated with the long-term Hospital for Special Surgery Score after TKA [27]. In synopsis of these findings, the evidence seems to be rare and conflicting.

The following limitations are acknowledged. This was a retrospective study with the weaknesses typically associated with such studies. We were not able to investigate for parameters other than those already available (WOMAC Score, ROM). It is regarded as significant limitation of the study that from 2093 TKA in the study period only 330 cases could be included in the analysis due to missing WOMAC or ROM data or due to the fact that other than the abovementioned CR implants had been used. A fact which may have led to selection bias. Another weakness was that ROM was not always measured by the same investigator. It was measured by physical therapists in the early postop phase and by physicians at the 1-year outpatient visit.

Its strength is that the current work generated scientific findings in a field where publications are very rare. Moreover, the findings obtained from the current work are based on case numbers far superior to those of previous research.

\section{Conclusions}

From the main findings that early postoperative ROM did not influence long-term knee score outcome or long-term ROM after TKA, it does not seem necessary to force ROM gain too strongly in the initial postoperative days. Probably, preoperative ROM and proper biomechanics of the TKA are predictors of much greater importance than is steepness of ROM ascent in the early postoperative period.

Acknowledgements This study was carried out with internal funds from Dept. of Orthopaedic Surgery, Medical University of Innsbruck, Innsbruck and Institute of Clinical Epidemiology, Tirol Kliniken, Innsbruck

Funding Open access funding provided by University of Innsbruck and Medical University of Innsbruck.. There is no funding source.
This study was supported by Dept. of Orthopaedic Surgery, Medical University of Innsbruck internal fund.

\section{Declarations}

Conflict of interest The authors declare that they have no conflict of interest. The authors alone are responsible for the content and writing of the article.

Ethical approval Any aspect of the work covered in this manuscript that has involved human patients has been conducted with the ethical approval (approval No. AN2016-0139) of all relevant bodies and such approvals are acknowledged within the manuscript.

Open Access This article is licensed under a Creative Commons Attribution 4.0 International License, which permits use, sharing, adaptation, distribution and reproduction in any medium or format, as long as you give appropriate credit to the original author(s) and the source, provide a link to the Creative Commons licence, and indicate if changes were made. The images or other third party material in this article are included in the article's Creative Commons licence, unless indicated otherwise in a credit line to the material. If material is not included in the article's Creative Commons licence and your intended use is not permitted by statutory regulation or exceeds the permitted use, you will need to obtain permission directly from the copyright holder. To view a copy of this licence, visit http://creativecommons.org/licenses/by/4.0/.

\section{References}

1. Australian hospital statistics 1995-96 A.I.o.H.a.W. (AIHW), Editor. (1997) AIHW Catalogue no. HSE 3: Canberra.

2. Australian hospital statistics 2007-08. (2009) AIHW Cat. no. HSE 71: Canberra.

3. Bade MJ et al (2014) Predicting functional performance and range of motion outcomes after total knee arthroplasty. Am J Phys Med Rehabil 93(7):579-585

4. van den Belt L et al (2015) Predictive factors of length of hospital stay after primary total knee arthroplasty. Knee Surg Sports TraumatolArthrosc 23(6): 1856-1862

5. Bin AbdRazak HR et al (2014) Total knee arthroplasty in Asian subjects: preoperative range of motion determines postoperative range of motion? OrthopSurg 6(1):33-37

6. Oldmeadow LB, McBurney H, Robertson VJ (2002) Hospital stay and discharge outcomes after knee arthroplasty: implications for physiotherapy practice. Aust J Physiother 48(2):117-121

7. Davies DM et al (2003) Effect of adjunctive range-of-motion therapy after primary total knee arthroplasty on the use of health services after hospital discharge. Can J Surg 46(1):30-36

8. Hewitt B, Shakespeare D (2001) Flexion vs. extension: a comparison of post-operative total knee arthroplasty mobilisation regimes. Knee 8(4):305-309

9. Kotani A, Yonekura A, Bourne RB (2005) Factors influencing range of motion after contemporary total knee arthroplasty. J Arthroplasty 20(7):850-856

10. Kamath AF et al (2010) Ethnic and gender differences in the functional disparities after primary total knee arthroplasty. ClinOrthopRelat Res 468(12):3355-3361

11. Kennedy DM et al (2008) Assessing recovery and establishing prognosis following total knee arthroplasty. PhysTher 88(1):22-32

12. Jones CA, Voaklander DC, Suarez-Alma ME (2003) Determinants of function after total knee arthroplasty. PhysTher 83(8):696-706 
13. Forrest GP, Roque JM, Dawodu ST (1999) Decreasing length of stay after total joint arthroplasty: effect on referrals to rehabilitation units. Arch Phys Med Rehabil 80(2):192-194

14. Ishii $\mathrm{Y}$ et al (2011) Prediction of range of motion 2 years after mobile-bearing total knee arthroplasty: PCL-retaining versus PCL-sacrificing. Knee Surg Sports TraumatolArthrosc 19(12):2002-2008

15. Ishii $Y$ et al (2008) Range of motion during the perioperative period in total knee arthroplasty. Arch Orthop Trauma Surg 128(8):795-799

16. Fisher DA et al (2007) Looks good but feels bad: factors that contribute to poor results after total knee arthroplasty. J Arthroplasty 22(6 Suppl 2):39-42

17. Naylor JM et al (2012) Is discharge knee range of motion a useful and relevant clinical indicator after total knee replacement? Part 2. J EvalClinPract 18(3):652-658

18. Stucki G et al (1996) Evaluation of a German version of WOMAC (Western Ontario and McMaster Universities) Arthrosis Index. Z Rheumatol 55(1):40-49

19. Mukaka MM (2012) Statistics corner: a guide to appropriate use of correlation coefficient in medical research. Malawi Med J 24(3):69-71

20. Yao D et al (2020) Manipulation under anesthesia as a therapy option for postoperative knee stiffness: a retrospective matchedpair analysis. Arch Orthop Trauma Surg 140(6):785-791

21. Hasegawa M, Takagita H, Sudo A (2015) Prediction of post-operative range of motion using intra-operative soft tissue balance in total knee arthroplasty with navigation. Comput Aided Surg 20(1):47-51

22 Ritter MA et al (2003) Predicting range of motion after total knee arthroplasty. Clustering, log-linear regression, and regression tree analysis. J Bone Joint Surg Am 85(7):1278-1285

23 Lam LO, Swift S, Shakespeare D (2003) Fixed flexion deformity and flexion after knee arthroplasty. What happens in the first 12 months after surgery and can a poor outcome be predicted? Knee 10(2): 181

24. Farahini $\mathrm{H}$ et al (2012) Factors influencing range of motion after total knee arthroplasty. Iran Red Crescent Med J 14(7):417-421

25. Sancheti KH et al (2013) Factors affecting range of motion in total knee arthroplasty using high flexion prosthesis: a prospective study. Indian J Orthop 47(1):50-56

26. Yanagisawa $S$ et al (2014) Influence of the anterior-posterior femoral translation on the range of motion in cruciate-retaining total knee arthroplasty. Knee Surg Sports TraumatolArthrosc 22(11):2709-2714

27. Zhang H, Cui L, Weng X (2016) Effect of preoperative factors on the clinical outcome of total knee arthroplasty. Zhonghua Yi Xue Za Zhi 96(17):1345-1348

Publisher's Note Springer Nature remains neutral with regard to jurisdictional claims in published maps and institutional affiliations. 